\title{
QUALITY OF LIFE ASSESSMENT AMONG CHILDREN AND ADOLE- SCENTS WITH CEREBRAL PALSY. AN OBSERVATIONAL DESCRIPTIVE STUDY IN THE CITY OF NAPLES AND ITS SURROUNDINGS
}

\author{
Dott. Marino Simone, Dott. Scuotto Luciana
}

KEYWORDS: quality of life, cerebral palsies in children

\section{ABSTRACT}

This study aims to assess the Quality of Life in children and adolescents with Cerebral Palsy in the city of Naples and its surroundings.

We have tried to photograph the QoL of subjects with cronic pathology in a specific social context and historic moment, and, if possible, delegate the priority areas of intervention and the actions for QoL improvement to other studies.

PedsQL 4.0- Generic Core ScaleTM was the main tool for the assessment of QoL in young subject with CP, which has been added to a personal data sheet.

The sample has been stratified according to the severity of disease as reported by the Gross Motor Function Classification System (from level I to V, in order of increasing severity), to age group as reported by PedsQL questionnaire (8-12 years and 13-18 years) and finally by gender.

The statistical analysis assesses the differences in the median values related to age, Physical Score, Psychosocial Score and Total Score, of all groups whose p value to the U Mann Whitney or Kruskal- Wallis test is less than 0.05. 27 patients, 13 males and 14 females, and their caregiver who are all mothers, were enrolled; of these 27 patients, 18 are between 8 and 12 years old and 9 are between 13 and 18 years old.

4 of these patients due to the presence of cognitive impairment were not able to answer the questionnaire, so only the information given by the caregivers were taken into account.

Referring to GMFCS, 11 patients are evalueted as level I, 5 patients as level II, 1 as III, 3 patients as IV and 7 patients as $V$.

Patients come from 5 Physical Rehabilitation centers in Naples and its surrounding.

Significant differences were found between patients from 8- 12 years and 13-18 years, where the adoloscents reported a drastic drop of Quality of Life ( $p=0.22$ on Total Score).

Furthermore, the initial hypothesis about the inverse proportionality between decrease in QoL and the severity of patology (evalueted with GMFCS) is confirmed, both from what reported by patients and their caregivers $(p=0.014$ in Total Score for both groups).

Finally it was found that the caregivers of patients with low severity of patologhy (level I and II) understimate the Quality of Life of their children, both in Physical funcitioning $(p=0.02)$ and in the Total Score $(p=0.04)$

The international literature agrees with result shown by this work and this allows us to ask question that can be shared even beyond the national borders.

Moreover, this small sample study can be a starting point for a future multicentric survey on regional territories, in order to allow an assessment of the Quality of Health assistance and to found any discrepancies.

\section{INTRODUCTION}

The Telos, to be intended in the Aristotelian way, of every social system and every health care system is to provide tools and solutions to subjects, citizens and patients, aimed to achieve their best well-being state. "The Republic safeguards health as a fundamental right of the individual and as a collective interest, and guarantees free medical care to the indigent", thus, not by chance, states the Article 32 of the Italian Constitution and in 1978 the insitution of the National Healthcare system tries to give a concrete meaning to this concept.

At the beginning of the third decade of the third millennium we must draw up an analysis of what has been done in these years verifying if we are really taking care of Italian citizens' health.

This research takes off from this premise, analyzing the Quality of Life in patients from 8 to 18 years old with Cerebral Palsy through the use of the PedsQLTM questionnaire, with the objective to understand if the health care system in the territories of Naples is really aimed at preserve their well-being and what kind of idea of well-being does exist. Analyzing in this subjects the Quality of Life related to this pathology has been a natural and consistent choice with the objective of this work, because it engages these patients during all their lives and needs a huge therapeutic attention, moreover this patology permeates inevitably 


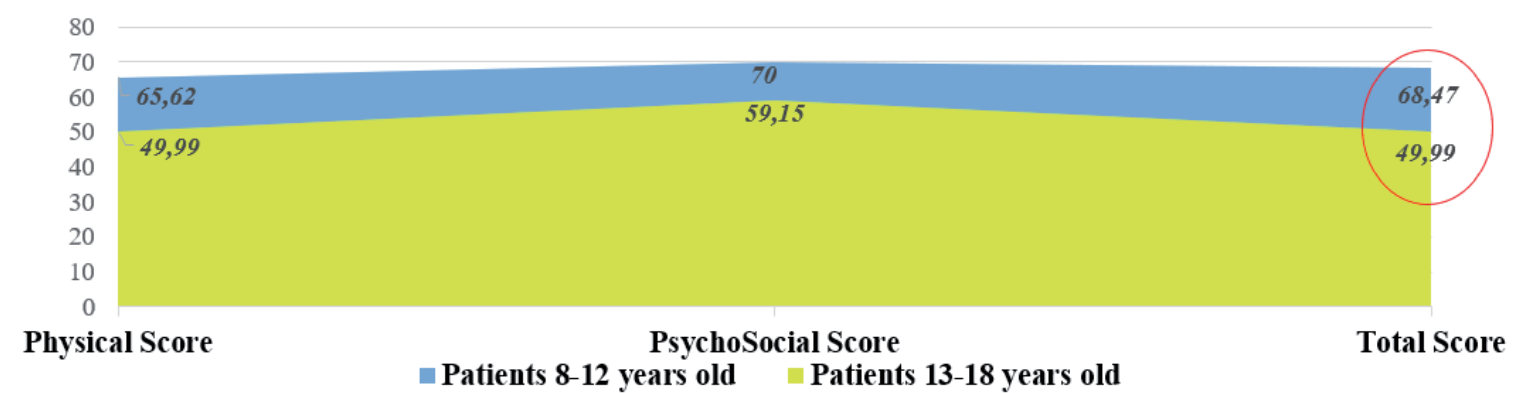

Graphic 1: gap in median values of three scores between patients from 8 to 12 years old and patients from 13 to 18 years old.

every aspect of their daily life.

Children who grow with this neurological disorder structures, like everyone else, their own conscience and their own idea of the world on the abilities with which he manages to explore it, but he will have to deal with a more complex and less coherent research than other people.

Their emotions, their relationship with their families and school and their world must be analyzed and interpreted in order to understand how and how much they can be helped.

My brief direct experience with children and adolescents with long-life-diseases, immediately showed me that attention restricted only to the components strictly related to the pathology, desineable from a rehabilitative point of view as functional, will shortly determine a saturation of the benefits perceivable by the patients, and this precisely because it is and will always be a partial intervention.

The only modalities that the operator has to overcome the relativity of his perspective and his intervention are, as in everyday life contexts, confrontation and interdisciplinarity, concepts that need to be accepted because they are coherent with the nature of our humanity.

\section{MATHERIALS AND METHODS}

For this observational study about Quality of Life, the PedsQLTM questionnaire was chosen and in particular the PedsQLTM 4.0 Generic Core Scale.

The PedsQLTMGeneric Core Scale questionnaire is self-administered and takes approximately 4 minutes to be completed.

The questionnaire is dedicated to subjects from 8 to 12 years old (preadolescents) and from 13 to 18 years old (adolescents), who differ each other only in the replacement of the term teens instead of kids.

It is a generic questionnaire that analyzes the Health Related Quality of Life, in fact investigates four domains related to physical (8 item), emotional (5 item), social (5 item) and scholastic (5 item) functioning in reference to the period of the last month and answers are provided on a Likert scale.

It is therefore possible to evaluate 3 types of scores that have been calculated according to the indications provided by the Mapi Research Trust:

- Physical Health Summary Score (PHSS): evaluates the only domain of physical functioning;

- Psychosocial Health Summary Score (PSHSS): evaluates the domains of emotional, social and scholastic functioning;

- Total Score (TS): evaluates Quality of Life con- sidering all four domains.

A higher score indicates a better Quality of Life.

The questionnaire consists of two sections, one compiled by the patients, and another one compiled by the caregiver which differ only in the use of the first and third person and are filled independently by the two subjects.

We used the Italian version of this questionnaire, translated by the Mapi Research Institute of Lyon.

In addition to the PedsQLTM questionnaire, was also used a Personal Data Sheet which analyzed the following information:

- $\quad$ For the adolescent: sex, age, class attended, residence, nationality and position occupied in the family (for exaple: first of two son).

- For the parent: sex, age, residence, educational level, nationality and if he is an adoptive parent or not.

Then it was delivered a release and an information sheet regarding the management and use of the collected data, all in a paper format and inserted in an envelope that was returned by the participants sealed, to ensure data protection.

For the statistical analysis of the data obtained was used Microsoft Excel, while the site www.socscistatistics.com was used for the calculation of the $U$ Mann-Whitney and Kruskal-Wallis tests.

First, were used U Mann-Whitney and Kruskal-Wallis tests, two non-parametric tests for ordinal variables in small samples $(\mathrm{n}<30)$, applied to the scores calculated by the test.

Pairs of groups, or even 3 groups, are compared and it was verified by these tests if they came from the same population or not, givent that this hypothesis is concretized for groups with $\mathrm{p}$ value less than 0.05 .

These statistical tests are performed for the three scores calculated from the total patient and relative caregivers test and, subsequently, this process is carried out for each group of patients and caregivers (separately) identified by three stratification modes: the first based on severity levels identified by the GMFCS, the second based on the two age groups already identified by the questionnaire (8-12 years old group and 13-18 years old group) and the third based on gender (male and female).

\section{RESULTS AND DISCUSSION}

From the analysis of the $p$ value made by the U MannWhitney and Kruskal-Wallis and by the median values, its clear that there are some groups whose median values deviate significantly.

The first two groups are composed by patients from 8 


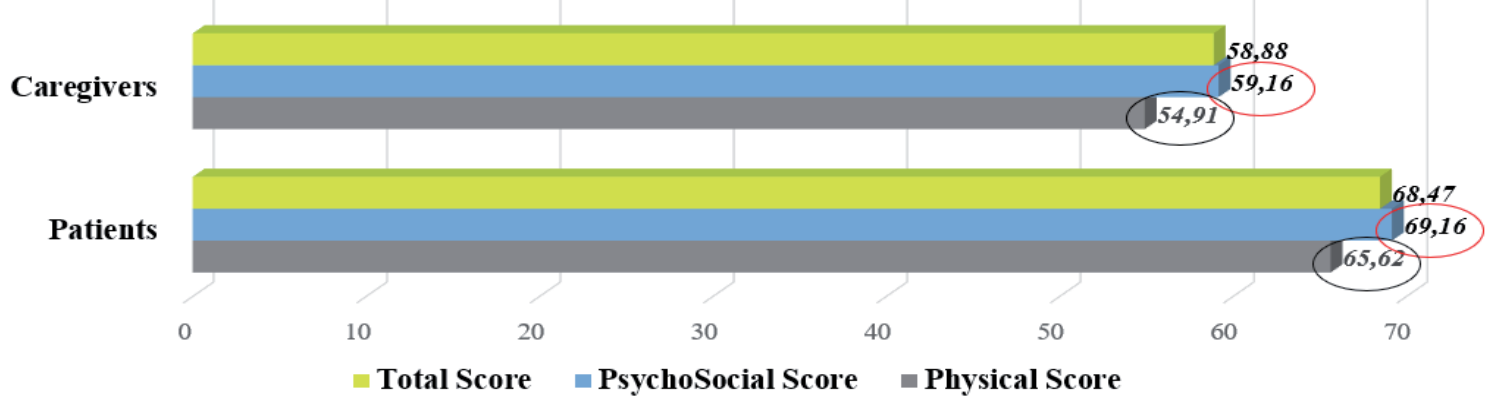

Graphic 2: gap in median values of the three scores between caregivers and patients with severity level of disease I and II- GMFCS

to 12 years old and by patients from 13 to 18 years old. In all 3 domains analyzed by the PedsQL questionnaire, a difference in median values can be seen, for the Physical Score (65.62 against 49.99 for the group of patients 13-18 years old), for the Psycho-social Score (70 against 59.16) and for the Total Score too (68.47 and 49.99), the only one in which the $p$ value goes below 0.05 .

These values, as shown in Graphic 1, lead us to believe that the Quality of Life in adolescent patients with Cerebral Palsy may be lower than that in children (or preadolescents).

Recent studies confirm that with increasing age the QoL in subjects with PC gradually decreases, although this phenomenon does not seem to be linked to an increase in the severity of the disease or to socio-demographic factors, but rather to a stressful relationship with parents.

Probably the relationship with parents is one of the reasons that can justify this phenomenon, but we must also consider how the need of autonomy can be for a teenager a crucial step for the development of his personality, and that could be the reason of the gap already shown; the prolongation of the dependency towards a parent, can somehow determine a dissatisfaction and frustration compaired to when it represented a physiological phase of development, was not perceived.

Some studies even reassure parents about the comparable QoL of children from 8 to 12 years old with PC to peers without the same disorder, and it is worrying to think that this overlap in adolescence goes astray.

Secondly, it can be seen that a higher severity of the pathology in terms of autonomy (GMFCS classification) determines a decrease of Quality of Life, and this is also detected by what parents express in their tests.

In fact, we see that the Quality of Life in subjects with PCI level I is higher than in subjects of level II, in the Physical Score (PHSS - 65.62 against 46.87), in the Psycho-social Score (PSHSS - 71, 66 against 60, the only score in which the p value exceeds 0.05$)$ and in the Total Score (71.91 and 51.08).

This phenomenon can also be found in the report of parents of patients with level I, level II and level V, where the relative median values shows a significant difference especially in the Physical and Total Score. The Gross Motor classification is in fact a good predictor of QoL especially for physical functioning, while it is less sensitive to detect variation of QoL in relation to the psycho-social domain, although some research shows that there is no correlation between Quality of Life and the severity level of pathology as descibed by this classification; however, even a topographical classification of the pathology seems to be directly related to the decreasing Quality of Life perceived by patients (worse QdV in the tetraplegic form than in the diplegic and hemiplegic ones).

Finally, a last significant result was found in the comparison between the groups of patients with CP' gravity level I and II and the relative caregivers, as expressed in graphic 2, because the difference in median values, espacially those to the Physical Score (65.62 and 54,91$)$ and the total score $(68.47$ and 58.88), combined with $\mathrm{p}$ values below 0.05 (0.02 for the PHSS and 0.04 for the TS), suggests how, especially in the presence of a not highly impairing disease, the perception of caregivers is that their children have a worse Quality of Life than what the children themselves really perceive.

A Finnish study carried out on 63 patients and 161 caregivers, as well as other studies conducted in recent years, agree that QoL reported in patient questionnaires is significantly higher than what is perceived by their caregivers, with the exception of Psycho-social Score.

Despite a shared point of view of the literature on this result, it may still be necessary for the health professional to give attention to the management of family dynamics in the presence of subjects with CP (or in other chronic diseases).

\section{REFERENCES}

1. Böling, S., \& Varho, T., \& Kiviranta, T., \& Haataja L. (2015). Quality of life of Finnish children with cerebral palsy. Disability and Rehabilitation, 38 (7), 683-688.

2. Büğüşan S., Kahraman A., \& Elbasan, B., \& Mutlu, A. (2017). Do adolescents with cerebral palsy agree with their caregivers on their participation and quality of life. Disability Health Journal, 11 (2), 287-292. 
3. Dickinson, H., \& Parkinson, K., \& Ravens-Sieberer, U., \& Schirripa, G., \& Thyen, U., \& Arnaud, C., et al. (2007). Self-reported quality of life of 8-12-year-old children with cerebral palsy: a cross-sectional European study. Lancet, 369 (9580), 2171- 2178 .

4. Livingston, M.H., \& Rosenbaum, P.L., \& Russell, D.J., \& Palisano, R.J. (2007). Quality of life among adolescents with cerebral palsy: what does the literature tell us? Developmental Medicine \& Child Neurology, 49 (3), $225-231$.

5. Longo, E., \& Badia, M., \& Begoña Orgaz, M., \& Gòmez- Vela, M. (2017). Comparing parent and child reports of health-related quality of life and their relationship with leisure participation in children and adolescents with Cerebral Palsy. Research in Developmental Disabilities, 71, 214- 222.

6. Omura, J., \& Fuentes, M., \& Bjornson, K. (2018). Participation in Daily Life: Influence on Quality of Life in Ambulatory Children with Cerebral Palsy. PM\&R, 10 (11), 1185- 1191.

7. Ozkan, Y. (2018). Child's quality of life and mother's burden in spastic cerebral palsy: a topographical classification perspective. Journal of International Medical Research, 46(8), 3131- 3137.

8. Pashmdarfard, M., \& Amini, M. (2017). Does Parent Report Gross Motor Function Level of Cerebral Palsy Children Impact on the Quality of Life in these Children? Iranian Journal of Child Neurology, 11(4), 52- 57.

9. Rapp, M., \& Eisemann, N., \& Arnaud, C., \& Ehlinger, V., \& Fauconnier, J., \& Marcelli, M. (2017). Predictors of parent-reported quality of life of adolescents with cerebral palsy: A longitudinal study. Research in Developmental Disabilities, 62, 259- 270.

10. Triassi, M. (2011). Il Management in Sanità - il governo dei sistemi sanitari complessi. Roma: Società Editrice Universo. 